\title{
Coexistence of ulcerative colitis and Sjögren's syndrome in a patient with Takayasu's arteritis and Hashimoto's thyroiditis
}

\author{
Hyun Woo Park ${ }^{1}$, Hyun Seok Lee ${ }^{1}$, Sejin Hwang ${ }^{1}$, Han Sol Lee ${ }^{1}$, Han-Ik Bae ${ }^{2}$, Ghilsuk Yoon ${ }^{2}$ \\ Departments of ${ }^{1}$ Internal Medicine and ${ }^{2}$ Pathology, Kyungpook National University School of Medicine, Daegu, Korea
}

A 31-year-old woman with a 15-year history of Takayasu's arteritis (TA) and a 13-year history of Hashimoto's thyroiditis presented with hematochezia. She received a diagnosis of Sjögren's syndrome at 1 month before her visit to Kyungpook National University Medical Center. Her colonoscopic findings were compatible with a diagnosis of ulcerative colitis (UC). She was treated with oral mesalazine, and her hematochezia symptoms subsequently disappeared. The coexistence of UC and TA has been reported; however, reports on the coexistence of UC and Sjögren's syndrome, or of UC and Hashimoto's thyroiditis are rare. Although the precise etiologies of these diseases are unknown, their presence together suggests that they may have a common pathophysiologic background. Furthermore, in patients with autoimmune or vascular diseases, including TA, systemic manifestations should be assessed with consideration of inflammatory bowel diseases including UC in the presence of gastrointestinal symptoms such as diarrhea and hematochezia. (Intest Res 2017;15:255-259)

Key Words: Takayasu arteritis; Colitis, ulcerative; Sjogren’s syndrome; Hashimoto disease

\section{INTRODUCTION}

Ulcerative colitis (UC) and Takayasu's arteritis (TA) are chronic inflammatory diseases with unknown etiologies, and their coexistence has been reported occasionally in the literature. ${ }^{1}$ Hashimoto's thyroiditis (HT) and Sjögren's syndrome (SS) are both inflammatory autoimmune diseases, and they have both been reported as being associated with UC in rare cases. $^{2,3}$ The likelihood of the simultaneous occurrence of these four diseases in a patient is considered extremely low.

Received November 11, 2015. Revised February 1, 2016. Accepted March 11, 2016. Published online March 7, 2017 Correspondence to Hyun Seok Lee, Department of Internal Medicine, Kyungpook National University Medical Center, Kyungpook National University School of Medicine, 807 Hoguk-ro, Buk-gu, Daegu 41404, Korea. Tel: +82-53-200-2603, Fax: +82-53-200-2027,E-mail: Ihsworld@nate.com

Financial support: None. Conflict of interest: None.

\section{CASE REPORT}

A 31-year-old woman visited gastroenterology outpatient clinic of Kyungpook National University Medical Center with a complaint of frequent hematochezia occurring for 1 month. She also complained of occasional periumbilical pain. Her medical history was as follows. At the age of 16 years, she was admitted to our hospital presenting with intermittent palpitations and dizziness, which she had been experiencing for 3 years. On physical examination, her resting blood pressure, as measured in her right upper arm, was $160 / 110 \mathrm{mmHg}$. However, her pulse at the radial artery on the left upper arm was not palpable, and bruits were audible over the left common carotid and left supraclavicular artery areas. Subacute or chronic TA was diagnosed with neck and chest CT angiography, which showed significant stenosis of the left subclavian artery and the proximal part of the left common carotid artery (Fig. 1). The patient was treated with acetylsalicylic acid to prevent an ischemic event. She also

\footnotetext{
๑ Copyright 2017. Korean Association for the Study of Intestinal Diseases. All rights reserved.

This is an Open Access article distributed under the terms of the Creative Commons Attribution Non-Commercial License (http://creativecommons.org/licenses/by-nc/4.0)

which permits unrestricted non-commercial use, distribution, and reproduction in any medium, provided the original work is properly cited.
} 


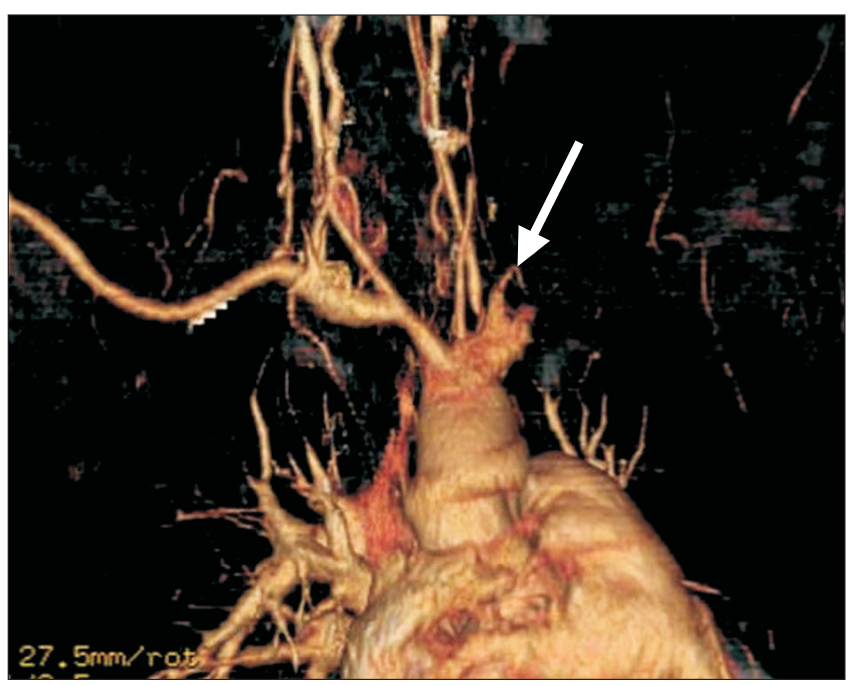

Fig. 1. Neck and chest CT angiography finding. CT angiography showing a narrowing of both the common carotid artery and a complete occlusion of the left subclavian vein (arrow) just after the branching of the vertebral artery.

received treatment with angioplasty of both renal arteries to control renovascular hypertension. At the age of 18 years, she presented with a progressive, painless goiter, and weight gain. Laboratory findings showed low levels of free T4 and T3, with elevated levels of antithyroid peroxidase antibodies, antithyroglobulin antibodies, and thyroid-stimulating hormones. Thyroid ultrasonography showed diffuse, enlarged heterogeneous thyroid glands without any focal lesions (Fig. 2). Color Doppler ultrasonography demonstrated mildly decreased parenchymal vascularity. These findings were consistent with HT, and the patient received hormone replacement with levothyroxine to treat her hypothyroidism.

One month before the visit described in this report, the patient consulted the department of rheumatology with a complaint of intermittent back pain, muscle ache, and dry mouth. She also had ocular pain and dry eyes for several years. Although laboratory findings showed negative results for anti-Ro/SSA and anti-La/SSB, and a positive result for ANA (1:80) with a positive rheumatoid factor, a labial salivary gland biopsy demonstrated a few lymphoplasma cells in the minor salivary gland (Fig. 3). On the basis of the histological findings, positive results of the Schirmer's test, "tear break-up time," and the clinical manifestation of characteristic tender points, SS with fibromyalgia was diagnosed, and the patient was treated with amitriptyline and hydroxychloroquine. She had no specific family history related to any of these diseases. During her physical examination, she had mild tenderness on her lower abdominal area. Laboratory

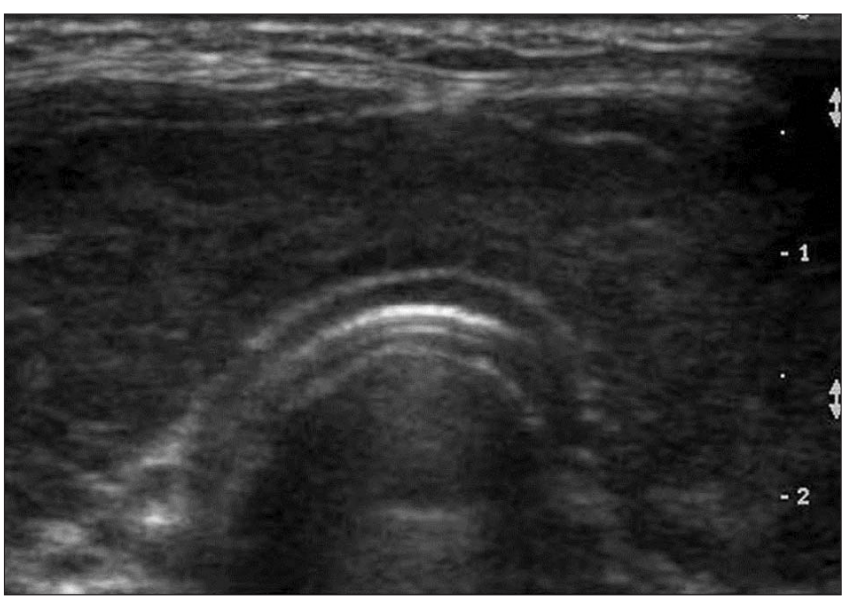

Fig. 2. Thyroid ultrasonotraphic finding. Ultrasonography showing a diffuse enlargement with heterogeneous echogenicity without any definite focal lesions of both thyroid glands.

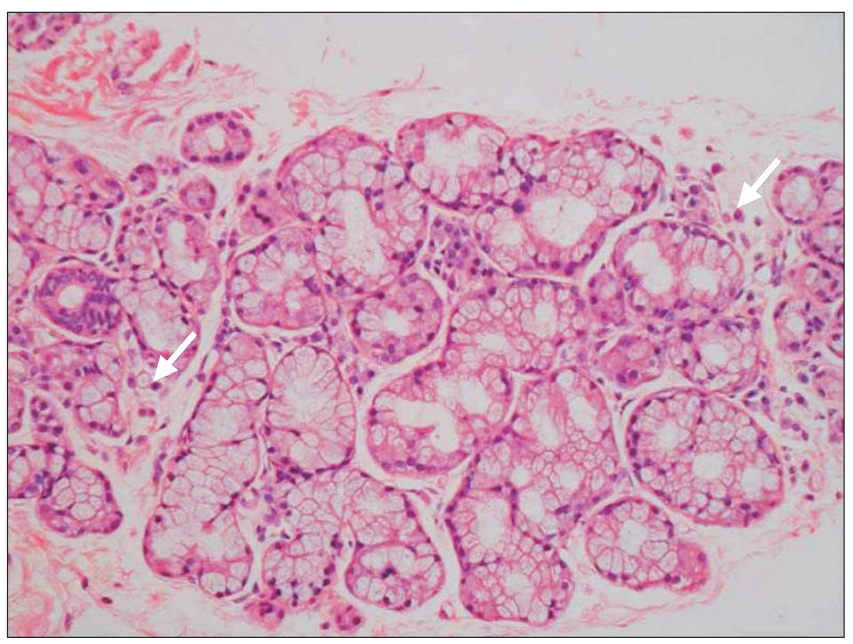

Fig. 3. Histopathological finding. Labial salivary gland biopsy exhibiting a few lymphoplasma cells (arrows) in the minor salivary gland ( $\mathrm{H} C \mathrm{E}$, $\times 200)$.

findings showed a white blood cell count of $6,540 / \mathrm{mm}^{3}$, hemoglobin level of $13.0 \mathrm{~g} / \mathrm{dL}$, platelet count of $296,000 / \mathrm{mm}^{3}$, ESR of $30 \mathrm{~mm} / \mathrm{hr}$ (reference range, $0-20 \mathrm{~mm} / \mathrm{hr}$ ), and serum high-sensitivity CRP level of $0.09 \mathrm{mg} / \mathrm{dL}$ (reference range, 0-0.5 mg/dL). An esophagogastroduodenoscopy revealed multiple erosions in the gastric antrum. A colonoscopy showed a decreased vascular pattern and erythematous mucosa with exudates that started in the rectum and extended to the cecum (Fig. 4A-C). Multiple biopsies were taken from the ascending, transverse, and sigmoid colons. Microscopic examinations of the biopsy specimens revealed moderately chronic active colitis with cryptitis, as well as crypt abscess, crypt distortion, and branching crypts without granuloma. 


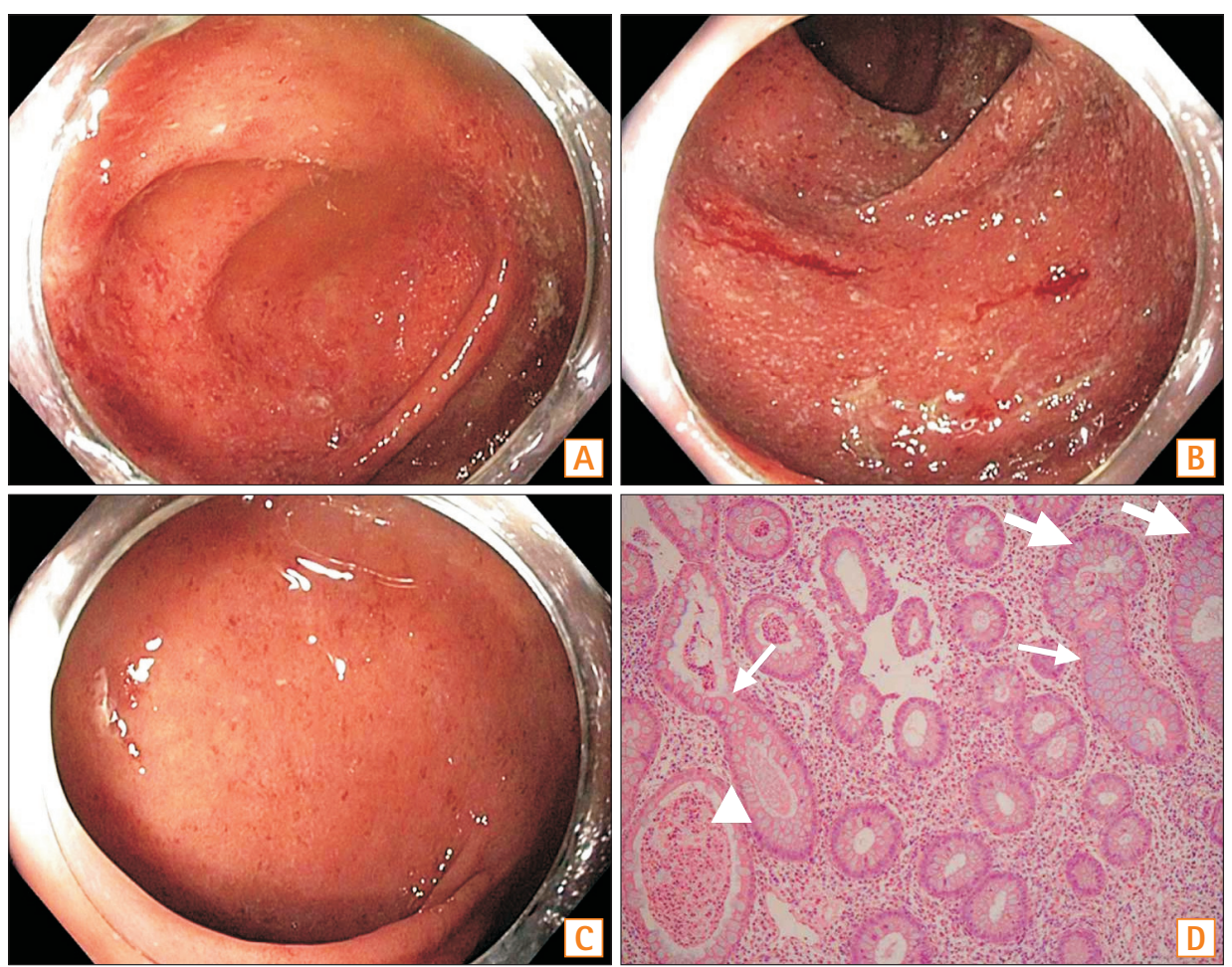

Fig. 4. Colonoscopic and histopathologic findings. (A-C) Colonoscopy showing decreased vascular patterns, and erythematous mucosa with exudates that started in the rectum and extended to the cecum. (D) Microscopic examination of biopsy specimens from the colon revealing moderately chronic active colitis with cryptitis, as well as crypt abscesses (arrowhead), crypt distortion (thin arrows), and branching crypts (thick arrows) $(\mathrm{HEEE}, \times 40)$.
The histological and colonoscopic findings were compatible with a diagnosis of UC (Fig. 4D). Colonoscopy also revealed a rectal polyp of approximately 9-mm size. The polyp was completely removed with endoscopic mucosal resection. The pathological examination of the polyp revealed a 9-mm neuroendocrine tumor confined to the mucosal and submucosal layers, with a clear deep resection margin and no lymphovascular invasion. An abdominal CT scan revealed an enhancement of the rectal mucosa. Additional laboratory findings were negative for antineutrophil cytoplasmic antibody (ANCA). The severity of UC was moderate, with a Mayo score of 6 . The patient received treatment with oral mesalazine $(3,000 \mathrm{mg} /$ day $)$, which resulted in the gradual disappearance of her hematochezia symptoms. No mucosal abnormality was observed on a follow-up colonoscopy, and neither metastasis nor recurrence of the neuroendocrine tumor was detected during a follow-up period of 2 years.

\section{DISCUSSION}

UC is an idiopathic chronic inflammatory disease that is limited to the mucosa and submucosa of the rectum and sigmoid colon, with the possibility of the lesions extending through the entire length of the colon. ${ }^{1}$ UC occurs among young adults, and is more frequent in Western than in Asian countries. Recently, the incidence of UC has been increasing in several Asian countries. ${ }^{4} \mathrm{UC}$ and $\mathrm{CD}$, the two major types of IBD, are sometimes related to other autoimmune diseases and have diverse extraintestinal symptoms, including vascular manifestations. Although some studies have shown that patients with UC and primary sclerosing cholangitis have a significantly higher risk for the development of colorectal neoplasias than do those with UC alone, the long-term outcomes of patients with UC coexisting with other autoimmune diseases have been reported only rarely. ${ }^{5}$ Some studies have suggested that there are associations between IBD and anti-inflammatory drugs such as aspirin and NSAIDs. ${ }^{6}$ The patient in our case had taken acetylsalicylic acid for the prevention of ischemic events beginning at the age of 16 years. She may also have taken NSAIDs for intermittent back pain and shoulder pain. However, a further study is needed to indicate whether these drugs affected the development of UC in this patient.

TA is a systemic vasculitis of undetermined etiology affecting the large arteries and large branches of the aorta, and most commonly occurs in young Asian women. The coexistence of UC and TA has been reported occasionally. As the specific HLA, including HLA-B52 and DR2, has been found in concurrent case reports of UC and TA, many studies have reported that genetic factors are important in the pathogenesis of these two diseases. ${ }^{8}$ A large-scale analysis of the genetic overlapping of TA and UC suggests that UC is a major com- 
plication of TA, and that these two diseases share a significant proportion of their genetic background, with HLA-B*52:01 possibly playing a role in their concurrence. ${ }^{7}$ Unfortunately, we could not conduct genetic testing in our patient. However, it is known that the presence of HLA-B52 is closely associated with TA in both Korean and Japanese patients. ${ }^{9}$

Serological markers such as ANCA and anti-Saccharomyces cerevisiae antibody (ASCA) are used to differentiate between UC and CD because of their relatively high specificities despite their low sensitivities. ${ }^{10}$ Some studies have reported that perinuclear ANCA and ASCA might be associated with more unfavorable outcomes for IBD patients, with ANCA positivity being reported as more frequent in UC when co-occurring with primary sclerosing cholangitis. ${ }^{11}$ Some rare cases of the coexistence of ANCA associated with vasculitis, such as microscopic polyangiitis and SS, or HT, have been reported. ${ }^{12}$ However, TA is known as ANCA paucivasculitis, and a study reported that serological markers such as ANCA and ASCA were not implacable when diagnosing IBD in patients with TA. ${ }^{13}$ In this study, the laboratory findings showed negative results for ANCA, and we did not perform testing for ASCA because of the distinct differentiation of UC from CD.

SS is an autoimmune disease causing chronic inflammation in the salivary and lacrimal glands, leading to hyposecretion. SS often affects organs other than the exocrine glands, and these extraglandular manifestations are observed in $25 \%$ of patients with SS. Among them, patients with SS accompanied with thyroid diseases have been reported with frequencies of $10 \%$ to $70 \%$, whereas patients with SS occurring with IBD have been reported very rarely. ${ }^{14}$ A report from Italy documented two patients with both UC and SS. Both patients were women, and in both instances, a diagnosis of UC preceded a diagnosis of SS by several days. ${ }^{15}$ However, another study on the prevalence of SS in patients with IBD revealed that the incidence of SS was not increased in patients with IBD compared with controls, indicating a lack of association between SS and IBD. ${ }^{2}$ Concomitant SS in IBD is difficult to understand, as the pathogenesis of this intestinal disorder is not yet clear and the current literature is conflicting. ${ }^{16}$

HT is the most common organ-specific autoimmune thyroid disease (ATD). ATDs are characterized by the presence of antibodies working against the thyroglobulin, thyroid peroxidase, or thyrotropin receptor autoantigens. The association between rheumatologic diseases and thyroid disorders has long been understood, with the most common being the association among rheumatoid arthritis, SS, and ATD. A study reported that the prevalence of SS among ATD patients was $14 \%$, and that $35 \%$ of the ATD patients studied were positive to ANA. ${ }^{3}$ In a study investigating the frequency of rheumatic diseases in patients with ATD, the most frequently associated disease was fibromyalgia, which was detected in $31 \%$ of the patients. ${ }^{17}$ Another study reported that $13.7 \%$ of ATD patients have had at least one autoimmune disease, most frequently lupus or SS. ${ }^{18}$

Polyautoimmunity was defined as the presence of more than one autoimmune disease in a single patient. A study investigated the incidence of polyimmunity in patients with systemic lupus erythematosus, rheumatoid arthritis, multiple sclerosis, and systemic sclerosis. In this study, the incidence of polyimmunity was observed in $34.4 \%$ of patients, and ATD and SS were the most frequent. ${ }^{19}$ When three or more autoimmune diseases coexist in a patient, the condition is known as multiple autoimmune syndrome (MAS). MAS can be classified into three types on the basis of the prevalence of coexisting diseases. Of the three types, our patient shared the most similarities with the description of type-3 MAS, which includes ATD, myasthenia gravis and/or thymoma, SS, pernicious anemia, idiopathic thrombocytopenic purpura, Addison's disease, type-1 diabetes mellitus, or vitiligo. However, the case did not entirely match the description of type-3 MAS in that the description does not include TA or UC. The exact pathogenesis of MAS is not established; however, environmental triggers in genetically susceptible persons are believed to be the cause of this immune regulation disorder. ${ }^{20}$

In this report, we examined a 31-year-old woman with TA and HT who developed UC and SS. The combination of these rare diseases in the patient suggests that they may have a common pathophysiologic background such as a genetic linkage. Therefore, we suggest further studies and long-term surveys to help identify other genetic factors and environmental agents that can contribute to the pathogenesis and coexistence of these rare diseases. Furthermore, we believe that it is important in cases of patients with autoimmune or vascular diseases including TA that their assessments of systemic manifestations take into consideration other autoimmune diseases and IBDs including UC in the presence of gastrointestinal symptoms such as diarrhea and hematochezia.

\section{REFERENCES}

1. Chae MJ, Yu CW, Lee SY, et al. Takayasu's disease in a patient with ulcerative colitis. Korean Circ J 2013;43:135-138. 
2. Palm Ø, Moum B, Gran JT. Estimation of Sjögren's syndrome among IBD patients: a six year post-diagnostic prevalence study. Scand J Rheumatol 2002;31:140-145.

3. Topal F, Senel E, Akbulut S, Topal F, Dölek Y. A new combination of multiple autoimmune syndrome? Coexistence of vitiligo, autoimmune thyroid disease and ulcerative colitis. Dermatol Reports 2011;3:e19. doi: 10.4081/dr.2011.e19.

4. Ng SC. Emerging leadership lecture. Inflammatory bowel disease in Asia: emergence of a "Western" disease. J Gastroenterol Hepatol 2015;30:440-445.

5. Soetikno RM, Lin OS, Heidenreich PA, Young HS, Blackstone MO. Increased risk of colorectal neoplasia in patients with primary sclerosing cholangitis and ulcerative colitis: a metaanalysis. Gastrointest Endosc 2002;56:48-54.

6. Ananthakrishnan AN, Higuchi LM, Huang ES, et al. Aspirin, nonsteroidal anti-inflammatory drug use, and risk for Crohn disease and ulcerative colitis: a cohort study. Ann Intern Med 2012;156:350-359.

7. Terao C, Matsumura T, Yoshifuji H, et al. Takayasu arteritis and ulcerative colitis: high rate of co-occurrence and genetic overlap. Arthritis Rheumatol 2015;67:2226-2232.

8. Takahashi N, Tanabe K, Sugamori T, et al. Association between Takayasu arteritis and ulcerative colitis: case report and review of serological HLA analysis. Med Sci Monit 2011;17:CS81-CS84.

9. Yajima M, Moriwaki R, Numano F, Park YB, Cho YD. Comparative studies between Japanese and Korean patients: comparison of the findings of angiography, HLA-Bw52, and clinical manifestations. Heart Vessels Suppl 1992;7:102-105.

10. Saibeni S, Folli C, de Franchis R, Borsi G, Vecchi M. Diagnostic role and clinical correlates of anti-Saccharomyces cerevisiae antibodies (ASCA) and anti-neutrophil cytoplasmic antibodies (p-ANCA) in Italian patients with inflammatory bowel diseases. Dig Liver Dis 2003;35:862-868.
11. Høie O, Aamodt G, Vermeire S, et al. Serological markers are associated with disease course in ulcerative colitis: a study in an unselected population-based cohort followed for 10 years. J Crohns Colitis 2008;2:114-122.

12. Ghorbel IB, Feki NB, Salem TB, et al. Microscopic polyangiitis associated with primary biliary cirrhosis, Sjogren's syndrome and Hashimoto's thyroiditis. Saudi J Kidney Dis Transpl 2015;26:359-362.

13. Ozbakir F, Ugurlu S, Celik AF, Seyahi E. Anti-Saccharomyces cerevisiae (ASCA) and anti-neutrophil cytoplasmic (ANCA) antibodies are not increased in Takayasu arteritis. Acta Reumatol Port 2011;36:20-23.

14. D’Arbonneau F, Ansart S, Le Berre R, Dueymes M, Youinou P, Pennec YL. Thyroid dysfunction in primary Sjögren's syndrome: a long-term followup study. Arthritis Rheum 2003;49:804-809.

15. Triantafillidis JK, Roussou P, Manousos ON, Dadioti P, Nicolakis D. Ulcerative colitis and Sjogren's syndrome in the same patient: report of two cases and a review of the literature. Ital J Gastroenterol 1994;26:299-302.

16. Katsanos KH, Saougos V, Kosmidou M, et al. Sjogren's syndrome in a patient with ulcerative colitis and primary sclerosing cholangitis: case report and review of the literature. J Crohns Colitis 2009;3:200-203.

17. Fraga A, Mintz G, Valle L, Flores-Izquierdo G. Takayasu's arteritis: frequency of systemic manifestations (study of 22 patients) and favorable response to maintenance steroid therapy with adrenocorticosteroids (12 patients). Arthritis Rheum 1972;15:617-624.

18. Cakir M, Samanci N, Balci N, Balci MK. Musculoskeletal manifestations in patients with thyroid disease. Clin Endocrinol (Oxf) 2003;59:162-167.

19. Rojas-Villarraga A, Amaya-Amaya J, Rodriguez-Rodriguez A, Mantilla RD, Anaya JM. Introducing polyautoimmunity: secondary autoimmune diseases no longer exist. Autoimmune Dis 2012;2012:254319.

20. Mohan MP, Ramesh TC. Multiple autoimmune syndrome. Indian J Dermatol Venereol Leprol 2003;69:298-299. 